\title{
A dynamic tire model based on HPSO-SVM
}

\author{
Yuexia Chen, Long Chen, Chen Huang, Ying Lu, Chen Wang \\ (School of Automotive and Traffic Engineering, Jiangsu University, Zhenjiang 212013, Jiangsu, China)
}

\begin{abstract}
In order to accurately describe the force mechanism of tires on agricultural roads and improve the life cycle of agricultural tires, a tire-deformable terrain model was established. The effects of tread pattern, wheel spine, tire sidewall elasticity, inflation pressure and soil deformation were considered in the model and fitted with a support vector machine (SVM) model. Hybrid particle swarm optimization (HPSO) was used to optimize the parameters of SVM prediction model, of which inertia weight and learning factor were improved. To verify the performance of the model, a tire force prediction model of agricultural vehicle with the improved SVM method was investigated, which was a complex nonlinear problem affected by many factors. Cross validation (CV) method was used to evaluate the training precision accuracy of the model, and then the improved HPSO was adopted to select parameters. Results showed that the choice randomness of specifying the parameters was avoided and the workload of the parameter selection was reduced. Compared with the dynamic tire model without considering the influence of tread pattern and wheel spine, the improved SVM model achieved a better prediction performance. The empirical results indicate that the HPSO based parameters optimization in SVM is feasible, which provides a practical guidance to tire force prediction of agricultural transport vehicles.
\end{abstract}

Keywords: agricultural vehicle, tire force prediction model, support vector machine, hybrid particle swarm optimization DOI: $10.25165 /$ j.ijabe.20191202.3227

Citation: Chen Y X, Chen L, Huang C, Lu Y, Wang C. A dynamic tire model based on HPSO-SVM. Int J Agric \& Biol Eng, 2019; 12(2): 36-41.

\section{Introduction}

As agricultural vehicle tire is one main source of the cost $\operatorname{losses}^{[1]}$, it is necessary to study the mechanical coupling mechanism of the tire to improve the tire life cycle of agricultural vehicles, to provide a reliable model basis for the follow-up research of intelligent agricultural vehicles, and to prepare for the introduction of advanced electronic control system ${ }^{[2]}$.

Recently, scholars from different countries have put forward tire models with different functions. The models can be divided into three categories: theoretical model, empirical model and semi-empirical model ${ }^{[3]}$. The theoretical model of the tire establishes a mathematical model describing the mechanical properties of the tire by simplifying the actual physical structure of the tire $^{[4]}$. However, theoretical models often have many simplifications, and their forms are complex and computationally inefficient, so it is difficult to apply them to agricultural vehicle $\operatorname{research}^{[5]}$. The empirical model is directly fitted to the tire test data under different conditions and has a good application basis ${ }^{[6]}$. However, due to the lack of theoretical basis, the model does not have the ability to extrapolate and predict. Also, as the working conditions of agricultural tires are complex and variable, the full

Received date: 2017-01-22 Accepted date: 2018-12-17

Biographies: Yuexia Chen, $\mathrm{PhD}$, Lecturer, research interests: vehicles integrated chassis control of vehicle dynamics, Email: cyx08@126.com; Chen Huang, PhD, Associate Professor, research interests: vehicles integrated chassis control of vehicle dynamics, Email: huangchen@ ujs.edu.cn; Ying Lu, $\mathrm{PhD}$, Associate Professor, research interests: automatic crash notification system and rear seat cushion rotation system, Email: luying@ujs.edu.cn; Chen Wang, MS, research interests: vehicles integrated chassis control of vehicle dynamics, Email: 18852861170@163.com.

*Corresponding author: Long Chen, $\mathrm{PhD}$, Professor, research interests: vehicles integrated chassis control of vehicle dynamics, School of Automotive and Traffic Engineering, Jiangsu University, 301 Xuefu Road, Zhenjiang 212013, Jiangsu, China. Tel: +86-511-88781835, Email: chenlong@ujs.edu.cn. amount of experimental work required to fully express the mechanical properties of the tires is extremely large $\mathrm{e}^{[7]}$. The semi-empirical model combines the advantages of the first two types of models, which features both high theoretical precision and good extrapolation ${ }^{[8]}$. It can describe some basic physical and structural characteristics of the tire, and also has high simulation accuracy, which is in good agreement with the experimental data ${ }^{[9]}$. But semi-empirical models typically treat the wheel as a rigid wheel, without regards to the filtering and hysteresis effects of the sidewall elasticity and inflation pressure and so on. It is mostly a steady-state model, and the tire-soil contact force cannot be calculated dynamically. Tong ${ }^{[10]}$ established an dynamic computing tire-soft pavement force model, but the role of tread pattern and wheel spine were not considered, which is a significant factor affecting tire stiffness. In addition, the representation coefficients of tire sidewall elasticity and inflation pressure were statically calculated which is changing in the actual operating conditions. Therefore, the combined effects of tread pattern, wheel spine, tire sidewall elasticity, inflation pressure are dynamically considered by using SVM to improve model accuracy in the paper.

SVM is a new statistical machine learning theory which converts the original nonlinear problems into linear problems in high dimensional feature space by nonlinear transformation ${ }^{[11,12]}$. SVM theory has been developing rapidly so far ${ }^{[13]}$. It has become a new method at the field of machine learning and data mining, which is mainly used for regression ${ }^{[14]}$ and classification ${ }^{[15]}$. It is very difficult for traditional SVM to accurately find the core of the parameters, and thus particle swarm optimization algorithm is applied to choosing parameters ${ }^{[16]}$. Nieto et al. ${ }^{[17]}$ proposed an online support vector machine optimized by improved particle swarm optimization, and Selakov et al. ${ }^{[18]}$ optimized the parameter selection of SVM by HPSO. But under larger predicting workload with dynamic condition, it couldn't keep the convergence speed and the accuracy of searching optimization. For this 
purpose, an improved HPSO is used to optimize parameters of SVM prediction model, and tire force prediction of agricultural vehicle based on HPSO-SVM is established considering dynamic prediction effect.

In this study, using cross-validation accuracy as the fitness function, the HPSO was used to optimize the parameters of SVM. The tire-deformable terrain model was developed based on HPSO-SVM, and the model performance was tested through simulation and experiment. A new idea for predicting tire force of agricultural vehicle was proposed.

\section{Dynamic tire model based on HPSO-SVM}

Considering that the working ground of agricultural vehicles is mostly soft road ${ }^{[19,20]}$, where tire stiffness is much greater than soil stiffness, tire deformation is relatively not obvious. Therefore, tire modeling under soft road conditions usually assumes that tire is a rigid wheel and only considers the deformation of soil. The dynamic tire model based on HPSO-SVM is composed of two parts: the ground tire model and the deformable terrain force model, as shown in Figure 1. This paper pays close attention to the ground tire model during the deformable terrain force model has already been covered thoroughly ${ }^{[4,5,7,10]}$.

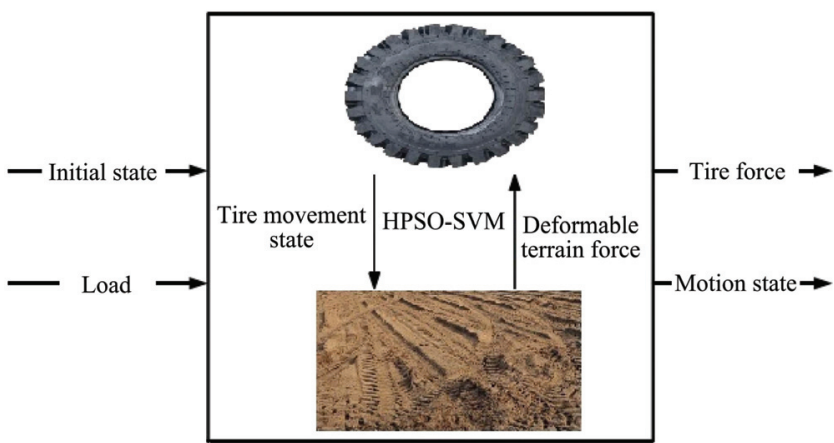

Figure 1 Dynamic tire model based on HPSO-SVM

According to the force of the tire, the ground tire model calculates the motion state of the tire, and transmits it to the deformable terrain force model, and then the deformable terrain force model dynamically calculates the tire-soil contact force, and finally transmits the contact force to the ground tire model. The HPSO-SVM model is used to dynamically describe tread pattern, wheel spine, tire sidewall elasticity and inflation pressure between ground tire model and deformable terrain force model.

\subsection{Ground tire model}

In this part, tire model is divided into three parts: rim, stiffness ring and spring damping system. The ring part represents the mass and inertia of rim and spoke in wheel, the stiffness ring represents the mass and inertia of tread part, and the spring damping system connects the rim part and the stiffness ring part to express the elasticity, inflatable pressure and tread pattern of the tire wall. The tire dynamics structure diagram is shown in Figure 2.

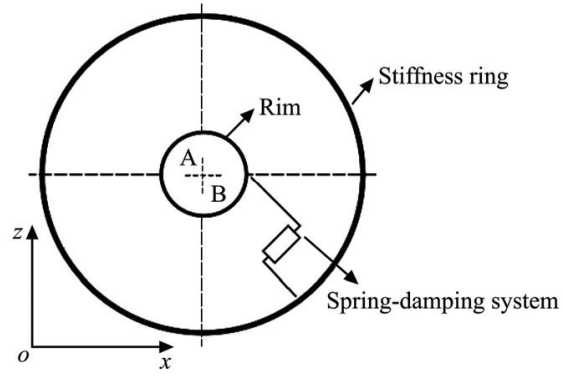

Figure 2 Tire dynamics structure
In the above figure, $\mathrm{A}$ and $\mathrm{B}$ are the center of mass of rim and stiffness ring respectively. The $x$-axis is located in the horizontal plane and the $z$-axis is vertical to the horizontal plane.

The rim is affected by spring damping force, gravity, wheel load and driving moment. According to Newton-Euler method, the dynamic equation of rim is as follows:

$$
\left\{\begin{array}{l}
m_{A} a_{A x}=F_{A x}+F_{x} \\
m_{A} a_{A z}=F_{A z}-m_{A} g+F_{z} \\
I_{A} c \&_{A}=M_{A}+M
\end{array}\right.
$$

where, $m_{A}$ is the rim mass; $a_{A x}$ and $a_{A z}, F_{A x}$ and $F_{A z}, F_{x}$ and $F_{z}$ are the components of the acceleration, spring damping force and axle load in the $X$ and $Z$ directions respectively; $I_{A}, \omega_{A}$ and $M_{A}$ are the moment of inertia, angular velocity, spring damping moment of the rim to its center of mass A respectively, and $M$ is the driving moment of the axle.

Stiffness ring is subjected to gravity, spring damping force and ground force. Its dynamic equation is as follows:

$$
\begin{gathered}
\left\{\begin{array}{l}
m_{B} a_{B x}=-F_{A x}+F_{g x} \\
m_{B} a_{B z}=-F_{A z}-m_{B} g+F_{g z} \\
I_{B}\left(\&_{B}=-M_{A}+M_{g}\right.
\end{array}\right. \\
\left\{\begin{array}{l}
F_{g x}=\int_{\theta_{2}}^{\theta_{1}} F_{\theta}(\theta) \cos \theta d \theta-\int_{\theta_{2}}^{\theta_{1}} F_{r}(\theta) \sin \theta d \theta \\
F_{g z}=\int_{\theta_{2}}^{\theta_{1}} F_{r}(\theta) \cos \theta d \theta+\int_{\theta_{2}}^{\theta_{1}} F_{\theta}(\theta) \sin \theta d \theta \\
M_{g}=R \int_{\theta_{2}}^{\theta_{1}} F_{\theta}(\theta) d \theta
\end{array}\right.
\end{gathered}
$$

where, $m_{B}$ is the stiffness ring mass; $a_{B x}, a_{B z}$ are the components of the acceleration in the $\mathrm{X}$ and $\mathrm{Z}$ direction; $I_{B}, \omega_{B}$ are the moment of inertia and angular velocity of the stiffness ring to its center of mass B respectively; $F_{g x}, F_{g z}$ are the components of the deformable terrain force in the $\mathrm{X}$ and $\mathrm{Z}$ direction; $M_{g}$ is the moment of the deformable terrain to the stiffness ring; $\theta, \theta_{1}, \theta_{2}$ are position angle, grounding angle and off-ground angle; $F_{\theta}(\theta), F_{r}(\theta)^{[10]}$ are the components of the stress of the contact soil unit at the position angle $\theta$ in the $\theta$ and $r$ direction, and $R$ is the wheel radius.

The spring damping force $F_{A}, M_{A}$ are consists of rotation angle $\theta_{A B}$, displacement $r_{A B}$, velocity $V_{A B}$ of stiffness ring relative to rim , and stiffness coefficient $k$, damping coefficient $c$, angular stiffness coefficient $k_{\theta}$ and angular damping coefficient $c_{\theta}$ of spring-damped system, which has strong non-linear characteristics because of the comprehensive effect of many factors. The multiple input multiple output model is established by HPSO-SVM as shown in Figure 3, and HPSO-SVM is established in section 2.2 to 2.4.

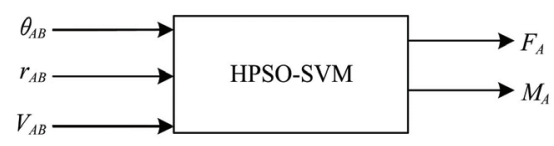

Figure 3 SVM spring damping model

\subsection{Least squares SVM model}

SVM is a Machine learning method based on statistical learning theory. Its core thoughts include both sample error and structural risk minimization, and thus has strong generalization ability and breakthrough the limitation of the data dimension. Least squares support vector machine (LSSVM) changes the inequality constraints in the basic SVM for the equality constraints.

Establish training sample set $\left\{\left(\boldsymbol{X}_{i}, \boldsymbol{Y}_{i}\right), i=1,2,3, \ldots, l\right\}$ where, $\boldsymbol{X}_{i} \in \mathrm{R}^{n}$ is the input column vector for the $i^{\text {th }}$ training sample, $\boldsymbol{Y}_{i} \in \mathrm{R}^{n}$ is the corresponding output values. These samples constitute model 


$$
\boldsymbol{Y}=f(\boldsymbol{X})=\boldsymbol{W} \boldsymbol{\varphi}(\boldsymbol{X})+\boldsymbol{b}=\left[\begin{array}{c}
\omega_{1}^{T} \boldsymbol{\varphi}(\boldsymbol{X})+b_{1} \\
\mathrm{M} \\
\omega_{n}^{T} \boldsymbol{\varphi}(\boldsymbol{X})+b_{n}
\end{array}\right]
$$

where, $\boldsymbol{\varphi}(\boldsymbol{X})$ is a mapping; $\boldsymbol{W}$ is the high dimensional feature space power matrix.

The optimal objective function of LSSVM is

$$
\min J(\boldsymbol{W}, e)=\frac{1}{2}\|\boldsymbol{W}\|^{2}+\frac{1}{2} \gamma \sum_{i=1}^{n} \sum_{j=1}^{l} e_{i j}^{2}
$$

where, $e_{i j}$ is the error between the model output and the sample, $\gamma$ is the penalty factor.

The constraint condition can be expressed as:

$$
\boldsymbol{Y}_{i j}=\boldsymbol{\omega}_{i}^{T} \boldsymbol{\varphi}\left(\boldsymbol{X}_{j}\right)+b_{i}+e_{i j}
$$

The LSSVM regression model is as follows:

$$
\boldsymbol{Y}(\boldsymbol{X})=\sum_{j=1}^{l} \alpha_{j} \boldsymbol{K}\left(\boldsymbol{X}, \boldsymbol{X}_{j}\right)+\boldsymbol{b}
$$

The radial basis kernel function is used in high dimensional space.

$$
\boldsymbol{K}\left(\boldsymbol{X}, \boldsymbol{X}_{j}\right)=\exp \left(-\frac{\left\|\boldsymbol{X}-\boldsymbol{X}_{j}\right\|^{2}}{2 \sigma^{2}}\right), \sigma>0
$$

where, $\sigma$ is the control parameter.

The model prediction effect is judged by

$$
E=\frac{1}{n} \sum_{i=1}^{n}\left(\hat{y}_{i}-y_{i}\right)^{2}
$$

\subsection{Hybrid particle swarm optimization algorithm}

Particle swarm optimization (PSO) algorithm is a heuristic algorithm, and it reflects the biological complex behavior, such as cooperation and competition among groups of swarm intelligence ${ }^{[21]}$. It has been applied to function optimization, neural network optimization, power system, combinatorial optimization etc.

Suppose in D dimension of search space, $\mathrm{m}$ particles are composed of a particle swarm, one of the space position of particles is $X_{i}=\left[x_{i 1}, x_{i 2}, \ldots, x_{i D}\right], i=1,2, \ldots, m$. Put it into optimization objective function and calculate the corresponding adaptive value. According to the fitness to measure the pros and cons of $X_{\mathrm{i}}$; the best positions of the particles is called individual historical best position, denoted as $P_{i}=\left[P_{i 1}, P_{i 2}, \ldots, P_{i D}\right]$. At the same time, each particle also has its own speed $V_{i}=\left[v_{i 1}, v_{i 2}, \ldots, v_{i D}\right]$. All particles has the best location which is called the global historical best position, denoted as:

$$
P_{g}=\left[p_{g 1}, p_{g 2}, \ldots, p_{i D}\right]
$$

The corresponding adaptive value is the best fitness value of global history. Each particle velocity and position update according to the following equation iteration:

$$
\left\{\begin{array}{l}
v_{i d}=w v_{i d}+c_{1} r_{1}\left(p_{i d}-x_{i d}\right)+c_{2} r_{2}\left(p_{\varepsilon d}-x_{i d}\right) \\
x_{i d}=x_{i d}+v_{i d}
\end{array}\right.
$$

where, $r_{1}, r_{2} \in[0,1]$ is the random numbers; the inertia weight $w$ is given by

$$
w=w_{\max }-\left(w_{\max }-w_{\min }\right) \times \frac{t_{\max }-t}{t_{\max }}
$$

where, $t$ is the number of iterations.

The acceleration coefficients $\mathrm{c}_{1}, \mathrm{c}_{2}$ are as follows ${ }^{[22]}$

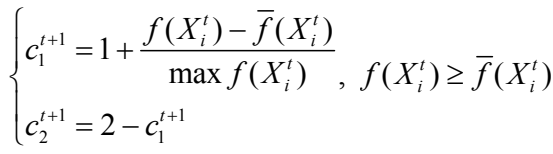

$$
\left\{\begin{array}{l}
c_{1}^{t+1}=1+\frac{\bar{f}\left(X_{i}^{t}\right)-f\left(X_{i}^{t}\right)}{f\left(X_{i}^{t}\right)-\min f\left(X_{i}^{t}\right)}, f\left(X_{i}^{t}\right)<\bar{f}\left(X_{i}^{t}\right) \\
c_{2}^{t+1}=2-c_{1}^{t+1}
\end{array}\right.
$$

where, $f\left(X_{i}^{t}\right)$ is the fitness of the $t^{\text {th }}$ iteration; $\bar{f}\left(X_{i}^{t}\right)$ is the average of $f\left(X_{i}^{t}\right)$.

For the particles which have no choice, crossover and mutation mechanism, the algorithm will not be able to search for other areas when a particle cluster around a local extremum and shows premature phenomenon. By using parallel population optimization, a hybrid particle swarm optimization method is put forward. The basic idea is as follows:

(1) The particle swarm is divided into two groups. One group is adopted to update population with Equation (11), and another group with the compression factor method.

(2) When two groups of particles appear precocious phenomenon, two groups of particles are crossed according to probability $P(P=0.5)$, and the maximum number of global extreme value in the two groups of particles is considered as the global extremum of the combined particle swarm.

(3) The combined population continues iterate, and update with the compression factor method. When there is precocious species, the half particles of larger fitness are maintained, and another half particles of smaller fitness are initialized again, thus algorithm convergence speed and optimization accuracy is not affected by variation of population.

\subsection{HPSO-SVM model}

The penalty factor $\gamma$ and kernel function parameter $\sigma$ play an important role in SVM prediction model, and the appropriate values tend to improve the prediction accuracy and reduce the workload. The improved hybrid particle swarm optimization algorithm is used to search the optimal parameters of SVM. Specific steps are as follows:

(1) Particle swarm initialization. The SVM parameters compose a particle $(\gamma, \sigma)$, the particle swarm is divided into two groups, and initial position and velocity is randomly generated, respectively.

(2) Fitness evaluation. Cross-Validation (CV) is used to test the performance of SVM model. The method is divide the raw data into $\mathrm{K}$ groups, with each separate subset data for a cross validation, and at the same time with the rest of the subset K-1 groups as the training set. Use the average $E$ of these $\mathrm{K}$ models final validation sets as the performance evaluation index.

(3) The renewal of individual optimal value $p_{\text {best }}$ and the global optimal value $g_{\text {best }}$. Two groups of particles update respectively according to the above the first step in the HPSO algorithm.

(4) Update the speed and position of particles.

(5) If both the two groups of particle swarm fitness value are less than set precision, the optimization is over and the optimal parameters is get by the smaller fitness value. If one group particle meets the accuracy requirement, the optimization is also over. Otherwise, iterate two groups of particle respectively, when 10 iterations do not change, go to the above the second step in the HPSO algorithm.

(6) Go to the third step in the HPSO algorithm, if the combined population meets the accuracy requirement, then it ends. Otherwise, continue iterating, and when 10 iterations do not change, introduce the mutation mechanism ${ }^{[23]}$ and go back to step (6). The detailed flow chart is shown in Figure 4. 


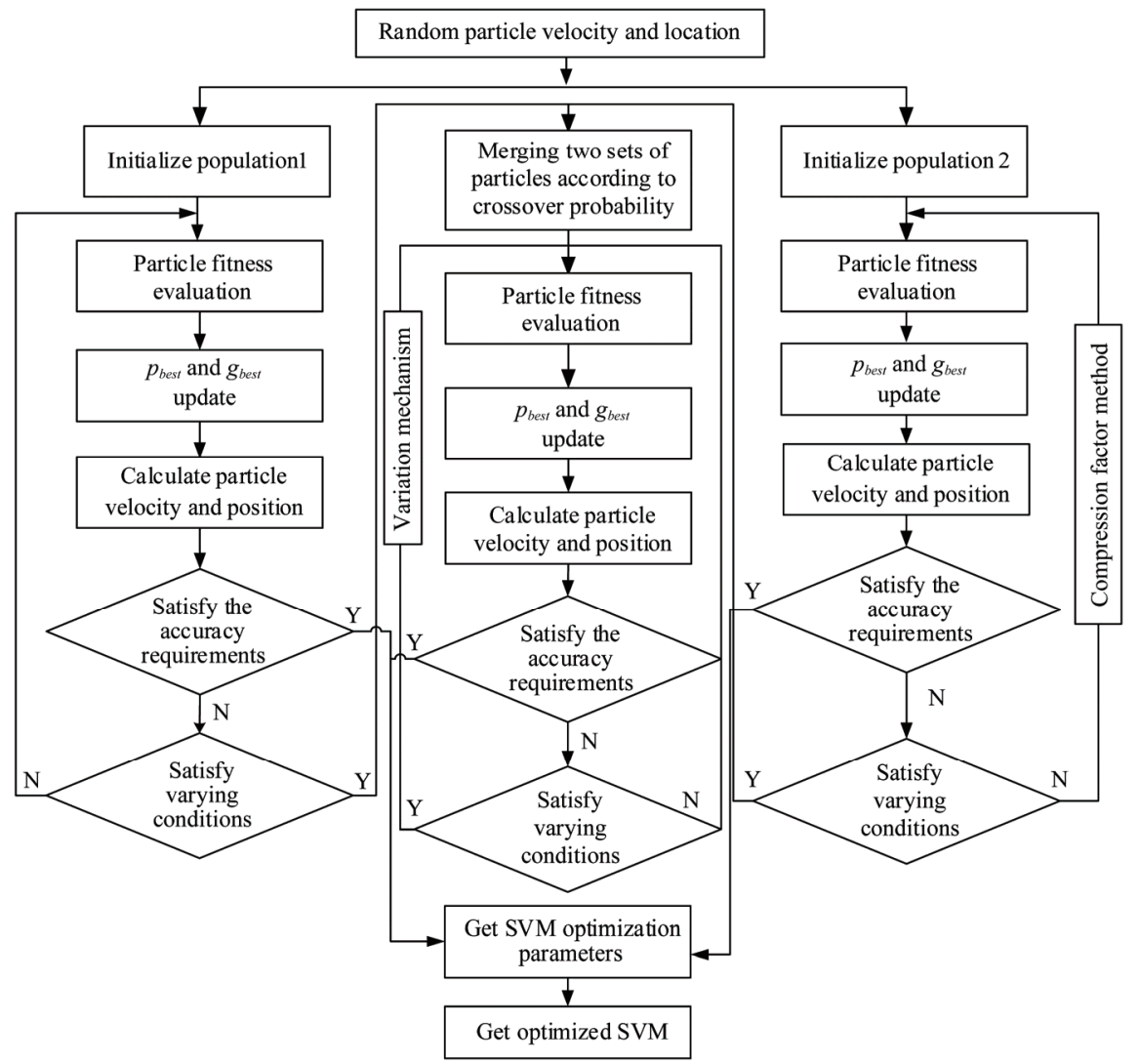

Figure 4 Flow chart of HPSO-SVM model

\section{Experiment and simulation}

\subsection{Simulation}

The effectiveness of the model is verified by simulation with MATLAB, and main steps of simulation are shown in Figure 5. The simulation data of [10] are used to validate the simulation performance, and the results are compared. The simulation parameters are set as shown in the Table 1. By simulation, the parameters in HPSO-SVM are set as follows: $w_{\max }=0.95, w_{\min }=0.3$, $c_{1}=1.35, c_{2}=1.45, t_{\max }=500, \gamma=487.81, \sigma=0.3642$.

\subsection{Simulation results and analysis}

Change rules of rolling resistance, drawbar pull and sinkage of wheel under different vertical loads and slip ratio are considered in the model. Figure 6 show the change rules of rolling resistance, drawbar pull under different vertical loads with fixing slip ratio $20 \%$.

As can be seen from Figure 6 above, rolling resistance and drawbar pull increase with increasing of the vertical load, and their values in dynamic tire model based on HPSO-SVM are slightly greater than that in dynamic model. This is consistent with the effect of tread pattern and wheel spine.

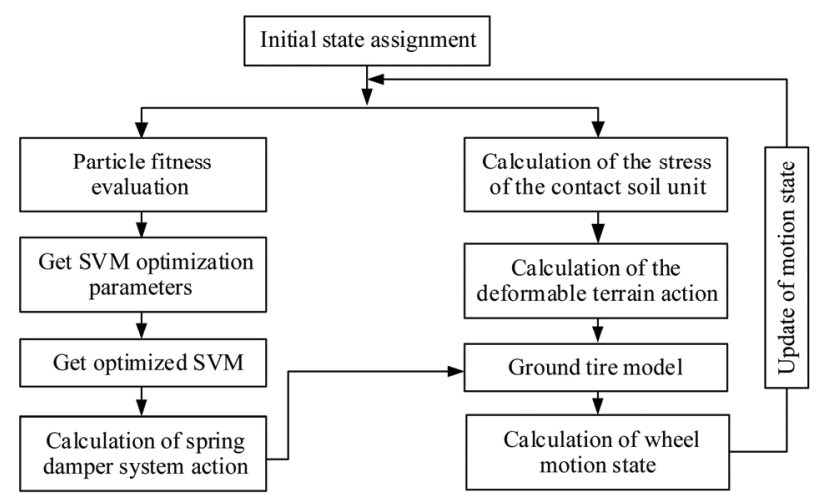

Figure 5 Flow chart of tire model
Table 1 Simulation parameters

\begin{tabular}{lcc}
\hline \multirow{2}{*}{ Paramete } & \multicolumn{2}{c}{ Value } \\
\cline { 2 - 3 } & $\begin{array}{c}\text { Dynamic tire } \\
\text { model }^{[10]}\end{array}$ & $\begin{array}{c}\text { HPSO-SVM } \\
\text { dynamic tire model }\end{array}$ \\
\hline Moment of inertia of rim $I_{A} / \mathrm{kg} \cdot \mathrm{m}^{-2}$ & 0.461 & 0.461 \\
Moment of inertia of stiffness ring $I_{B} / \mathrm{kg} \cdot \mathrm{m}^{-2}$ & 0.809 & 0.809 \\
Tire width $d / \mathrm{m}$ & 0.235 & 0.235 \\
Nominal tire load/N & 4420 & 4420 \\
Free radius $R / \mathrm{m}$ & 0.35785 & 0.35785 \\
Mass of rim $m_{A} / \mathrm{kg}$ & 3.2 & 3.2 \\
Mass of stiffness ring $m_{B} / \mathrm{kg}$ & 9.6 & 9.6 \\
Longitudinal stiffness coefficient $k_{x} / \mathrm{N} \cdot \mathrm{m}^{-1}$ & $2.86 \times 10^{5}$ & $/$ \\
Longitudinal damping coefficient $c_{x} / \mathrm{N} \cdot \mathrm{m}^{-1} \cdot \mathrm{s}^{-1}$ & $1.4 \times 10^{4}$ & $/$ \\
Vertical stiffness coefficient $k_{z} / \mathrm{N} \cdot \mathrm{m}^{-1}$ & $2.51 \times 10^{5}$ & $/$ \\
Vertical damping coefficient $c_{z} / \mathrm{N} \cdot \mathrm{m}^{-1} \cdot \mathrm{s}^{-1}$ & $1.26 \times 10^{4}$ & $/$ \\
Angular stiffness coefficient $k_{\theta} / \mathrm{N} \cdot \mathrm{rad}^{-1}$ & $3.49 \times 10^{4}$ & $/$ \\
Angular damping coefficient $c_{\theta} / \mathrm{N} \cdot \mathrm{rad}^{-1} \cdot \mathrm{s}^{-1}$ & $1.73 \times 10^{3}$ & $/$ \\
\hline
\end{tabular}

The picture 7 shows change rule of sinkage of wheel under different vertical loads with fixing slip ratio $20 \%$. The two models have the same law of change. The sinkage increases with the increase of vertical load, but the increasing range decreases gradually. The sinkage of the model in this paper is less than that of the dynamic model because of the influence of tread pattern and wheel spine.

Changing trend of rolling resistance and drawbar pull under different slip ratios with fixing vertical load $4000 \mathrm{~N}$ are shown in Figure 8.

It can be seen from the figure 8 that with the changing trend of rolling resistance and drawbar pull are the same between the two models. Compared with the dynamic tire model, the amplitude of variation in the HPSO-SVM tire model are larger. 


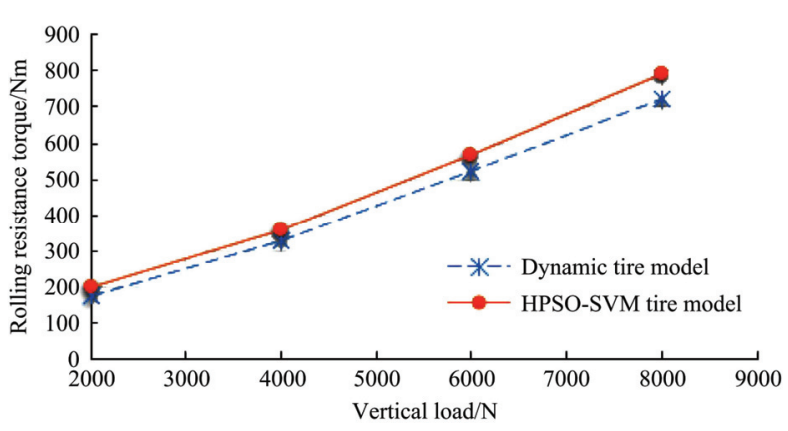

a. Rolling resistance torque

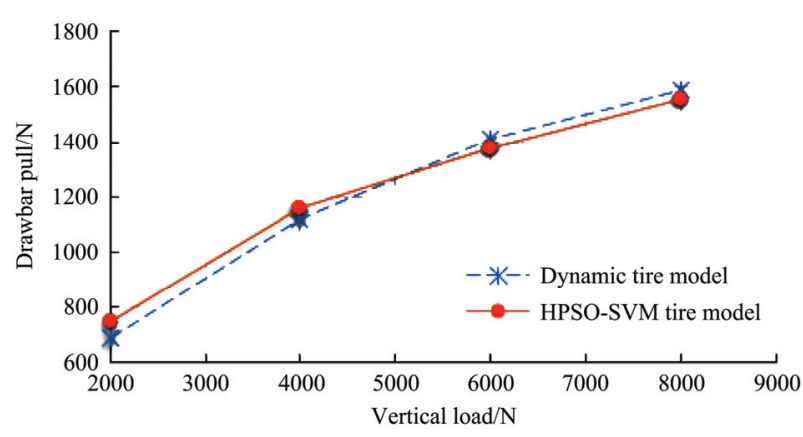

b. Drawbar pull

Figure 6 Change rules of rolling resistance and drawbar pull under different loads

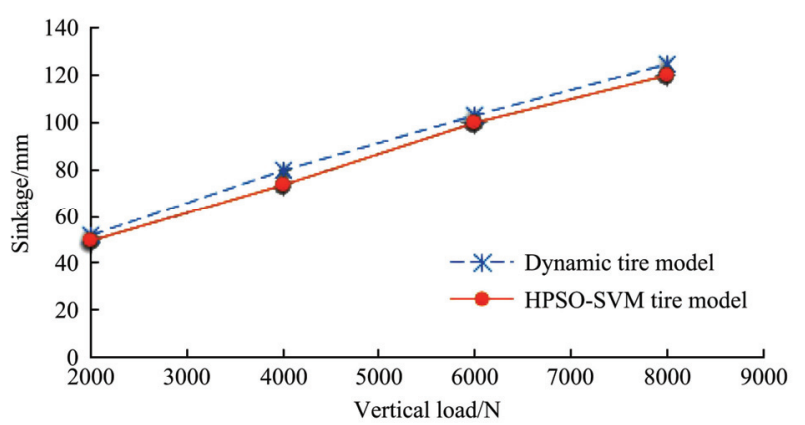

Figure 7 Change rules of sinkage of the wheel under different loads

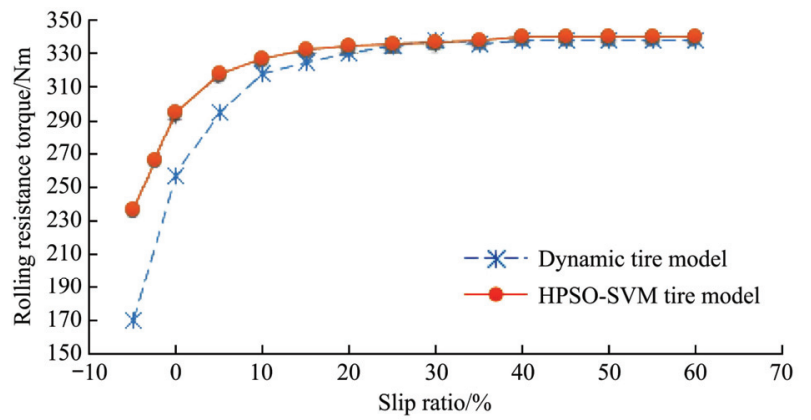

a. Rolling resistance torque

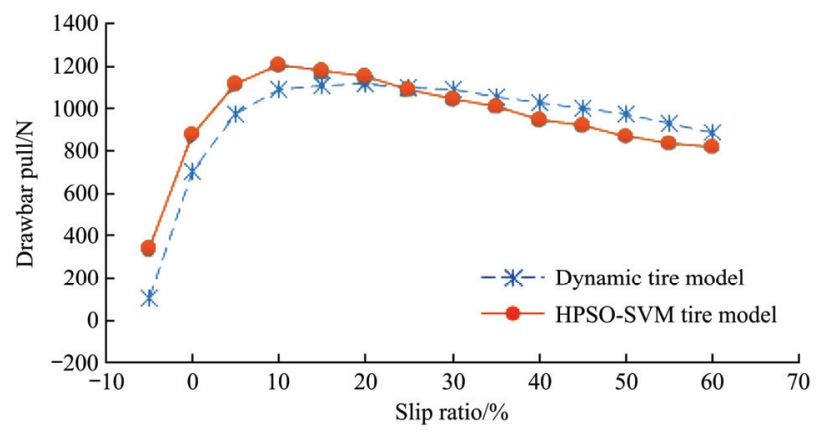

b. Drawbar pull

Figure 8 Change rules of rolling resistance and drawbar pull under different slip ratios

\subsection{Experiments}

The model was verified by the experimental data in [24]. The comparison results between simulation and experiment are shown in the Figures 9-11.

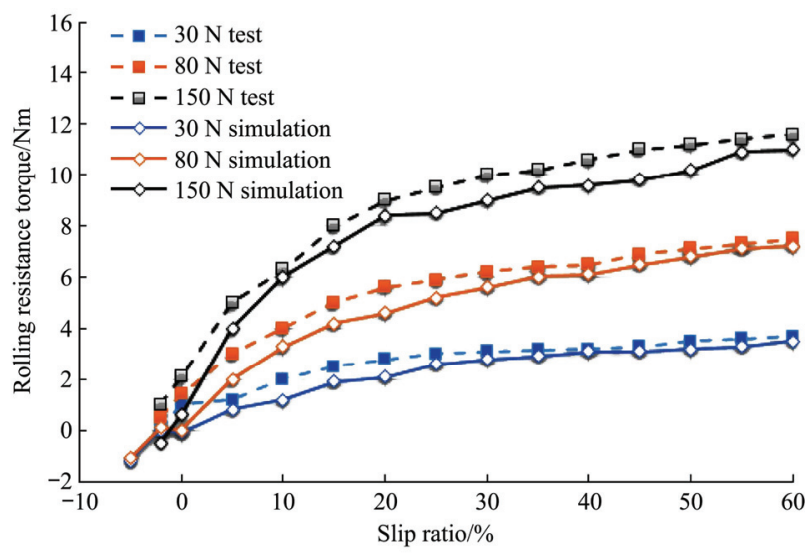

Figure 9 Rolling resistance under different slip ratios

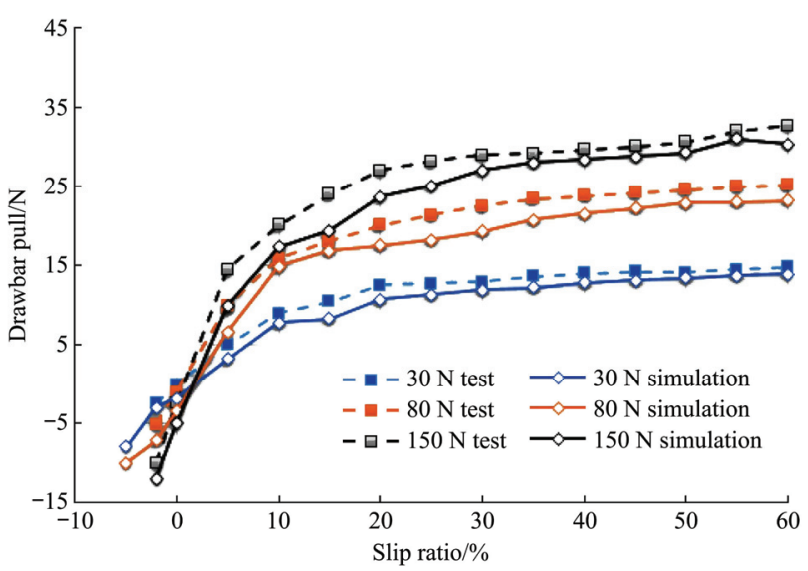

Figure 10 Drawbar pull under different slip ratios

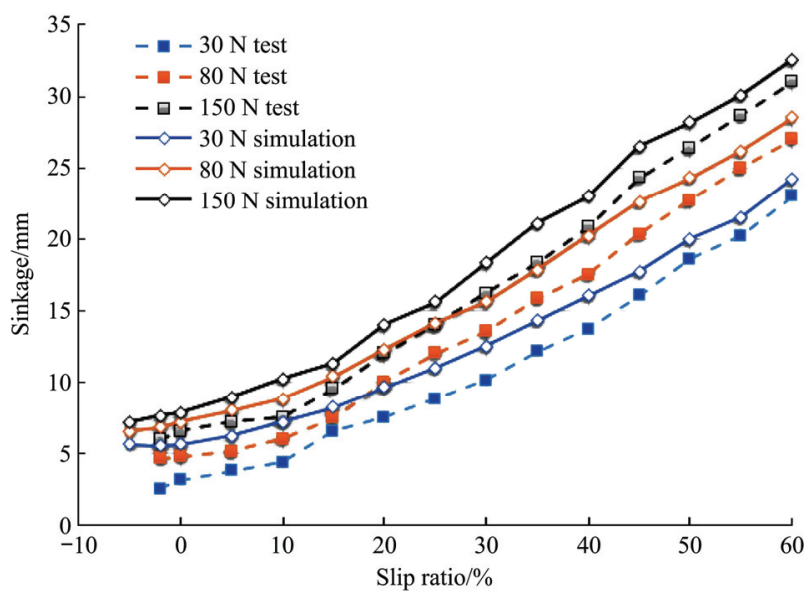

Figure 11 Sinkage under different slip ratios

Contrast results from the above figures show that although there are slight differences between the measured values and the starting point, the trends of simulation and experiment are consistent. The sinkage of the test is less than the simulation value, while the rolling resistance and the drawbar pull are slightly larger than it. This may be due to the failure to consider the tire roll deformation.

\section{Conclusions}

An improved HPSO was proposed, which introduces the multi population parallel optimization and cross mutation mechanism to 
improve the population structure, and solves the contradiction between improving the searching ability and accelerating the convergence speed in SVM model.

The improved dynamic tire model based on HPSO-SVM was investigated to predict tire power of agricultural vehicles. The tires force prediction model based on HPSO-SVM algorithm was feasible in simulated experiment verification. In contrast with dynamic tire model, the improved HPSO-SVM model had simpler modeling process and higher fitting accuracy, which could solve the nonlinear problem of tire characteristics better.

Utilizing the improved HPSO-SVM model, the future direction of the research will be further analysis of tire cornering characteristics affected by road, the tire structure, tire load, speed, lateral acceleration, and a more complex nonlinear tire cornering characteristics model will be constructed, in order to account for control system's analysis and synthesis.

\section{Acknowledgements}

We acknowledge that this project financially supported by the National Natural Science Foundation of China (Grant No. U1564201, 51605195, 51605197, 51875255), Jiangsu Provincial Natural Science Foundation of China (Grant No. BK20160524).

\section{[References]}

[1] Zou X, Wang T, Hao L. How to properly use and maintain tires for agricultural vehicles. Farm Machinery Using \& Maintenance, 2015; 3: 58-58. (in Chinese)

[2] Chen Y, Chen L, Wang R, Xu X, Shen Y, Liu Y. Modeling and test on height adjustment system of electrically-controlled air suspension for agricultural vehicles. Int J Agric \& Biol Eng, 2016; 9(2): 40-47.

[3] Guo K. UniTire: Unified tire model. Journal of Mechanical Engineering, 2016; 52(12): 90-99.

[4] Cueto O G, Coronel C E I, Bravo E L, Morfa C R, Suárez M H. Modelling in FEM the soil pressures distribution caused by a tyre on a Rhodic Ferralsol soil. Journal of Terramechanics, 2016; 63: 61-67.

[5] Zhao C, Zang M. Analysis of rigid tire traction performance on a sandy soil by $3 \mathrm{D}$ finite element-discrete element method. Journal of Terramechanics, 2014; 55(7): 29-37.

[6] Spentzas K N, Alkhazali I, Demic M. Dynamics of four-wheel-steering vehicles. Forschung Im Ingenieurwesen, 2001; 66(6): 260-266.

[7] Grisso, R, Perumpral J, Zoz F. An empirical model for tractive performance of rubber-tracks in agricultural soils. Journal of Terramechanics, 2006; 43(2): 225-236.

[8] Witzel P. The Hohenheim Tyre Model: A validated approach for the simulation of high volume tyres - Part I: Model structure and parameterisation. Journal of Terramechanics, 2017; 75: 3-14.

[9] Vu T D, Duhamel D, Abbadi Z, Yin H P, Gaudin A. A nonlinear circular ring model with rotating effects for tire vibrations. Journal of Sound \& Vibration, 2017; 388: 245-271.

[10] Tong K. Study on dynamic interaction model between tire and deformable terrain, Jilin University, 2017. (in Chinese)

[11] Li D, Ju Y, Zou Q. Protein Folds Prediction with Hierarchical Structured SVM. Current Proteomics, 2016; 13(2): 79-85.

[12] Chen Y, Chen L, Xu X, Wang R, Yang X. Chaotic Motion in a Nonlinear Car Model Excited by Multi-frequency Road Surface Profile. Chinese Journal of Mechanical Engineering, 2017; 30(3): 689-697.

[13] López J, Maldonado S, Carrasco M. A novel multi-class SVM model using second-order cone constraints. Applied Intelligence, 2016; 2: 1-13.

[14] Yoon H, Hyun Y, Ha K, Lee K K, Kim G B. A method to improve the stability and accuracy of ANN- and SVM-based time series models for long-term groundwater level predictions. Computers \& Geosciences, 2016; 90: 144-155.

[15] Chen Y, Chen L, Zha Q, Jing P, Xie J, Xiong X. Forecasting model of travel mode based on latent variable SVM. Journal of Southeast University, 2016; 46(6): 1314-1317.

[16] Long Y, Du Z J, Wang W D, Zhao G Y, Xu G Q, He L, et al. PSO-SVM-based online locomotion mode identification for rehabilitation robotic exoskeletons. Sensors, 2016; 16(9): 1408.

[17] García Nieto P J, García-Gonzalo E, Sánchez Lasheras F, Cos Juez F J. Hybrid PSO-SVM-based method for forecasting of the remaining useful life for aircraft engines and evaluation of its reliability. Reliability Engineering \& System Safety, 2015; 138: 219-231.

[18] Selakov A, Cvijetinović D, Milović L, Mellon S, Bekut D. Hybrid PSO-SVM method for short-term load forecasting during periods with significant temperature variations in city of Burbank. Applied Soft Computing, 2014; 16(3): 80-88.

[19] Guan X, Yang P, Li L, Tan Y. Multifractal characteristic of soil pore distribution after long-term recycled water irrigation. Journal of Drainage and Irrigation Machinery Engineering, 2018; 36(11): 1163-1167. (in Chinese)

[20] Xu C, Hou S, Yao Z, Zhang F, She D. Spatial variability and scale effect of soil bulk density of farm land in South China. Journal of Drainage and Irrigation Machinery Engineering, 2017; 35(5): 424-429. (in Chinese)

[21] Ma R, Wei Z, Chen X, Ma S. Barbed labyrinth channel optimization based on constrained multi-objective particle swarm algorithm. Journal of Drainage and Irrigation Machinery Engineering, 2018; 36(12): 1330-1336. (in Chinese)

[22] Wang F Z, Shi X L. Tap-changer fault diagnosis of transformer based on improved PSO-SVM. Electronic Measurement Technology, 2016; 39(11): 190-194. (in Chinese)

[23] Frans V D B. An analysis of particle swarm optimizers. University of Pretoria, 2002.

[24] Ding L, Gao H, Deng Z, Nagatani K, Yoshida K. Experimental study and analysis on driving wheels' performance for planetary exploration rovers moving in deformable soil. Journal of Terramechanics, 2011; 48(1): $27-45$. 